\title{
Pedestrian flow characteristics through bends: Effects of angle and desired speed
}

\author{
Jiahua Zhang1, Charitha Dias' ${ }^{2}$, Majid Sarvi $^{3}$, Miho Iryo-Asano ${ }^{4}$ \\ ${ }^{1,2}$ Institute of Industrial Science/The University of Tokyo \\ 4-6-1 Komaba, Meguro-ku, Tokyo, Japan \\ zhangjh@iis.u-tokyo.ac.jp; cdias@iiis.u-tokyo.ac.jp \\ ${ }^{3}$ Department of Infrastructure Engineering/The University of Melbourne \\ Building 176, Victoria 3010, Melbourne, Australia \\ majid.sarvi@unimelb.edu.au \\ ${ }^{4}$ Graduate School of Environmental Studies/Nagoya University \\ Environmental Studies Hall, Furo-cho, Chikusa-ku, Nagoya, Japan \\ iryo@nagoya-u.jp
}

\begin{abstract}
This study quantitatively described how the desired speed, which may reflect the emergency level, and the angle of bend affect the pedestrian flow by comparing fundamental diagrams derived from trajectory data collected through laboratory experiments. Results showed that the slow running $(\approx 2.8 \mathrm{~m} / \mathrm{s}$ speed) can increase the maximum flow through a bend by around $60 \%$ compared to normal walking $(\approx 1.4 \mathrm{~m} / \mathrm{s}$ speed) regardless of the turning angle. Further, it was found that the turning angle of the bend has a stronger negative impact on the moving speed of crowds under running conditions. Compared to the turning angle, congestion level seemed to have a minor impact on the average moving speed through the bends. On the other hand, for $90^{\circ}$ and $180^{\circ}$ bends, the variations of the speed were observed to decrease with the increase of density which indicated that although congestion level deteriorated the flow conditions at bends, it homogenized the collective moving speed of pedestrians.
\end{abstract}

Keywords: Pedestrian flow, Pedestrian turning behaviour, Fundamental diagram, Crowd experiment

\section{Introduction}

As experimentally verified in previous studies [1-2], corridors with bends are inefficient for crowd movements and could become bottlenecks under emergency as well as normal conditions. Therefore, when public spaces are planned and designed, attention should be paid to such locations. As a first step, this study aims to quantitatively describe how the desired speed and the angle of bend affect the pedestrian flow characteristics by comparing fundamental diagrams derived from trajectory data. On one hand, the measurement results from this experiment could be used to estimate the capacity of bends with different angles under different situations (i.e., normal or evacuation); on the other hand, the findings from this study could also be beneficial for the validation and calibration of crowd simulation models.

\section{Experiment Setup}

The data used for this study were collected from an experiment conducted at Monash University in October 2013 [2]. Trajectory data for walking through a straight $\left(0^{\circ}\right)$ and different angled $\left(45^{\circ}, 90^{\circ}\right.$ and $\left.180^{\circ}\right)$ corridors (corridor width is $1.5 \mathrm{~m}$ ) were collected under two different desired speed levels, i.e., normal speed walking (individual speed $\approx 1.4 \mathrm{~m} / \mathrm{s}$ ) and slow speed running (individual speed $\approx 2.8 \mathrm{~m} / \mathrm{s}$ ). Around 55 individuals participated in this experiment and each experiment scenario was repeated 3 times. Initial conditions were kept similar for all these experiments. Fig. 1 shows the experiment setup and a snapshot during one experiment scenario. The positions of each pedestrian's head were manually tracked at $0.12 \mathrm{~s}$ intervals from video recordings to obtain trajectories of individuals. 

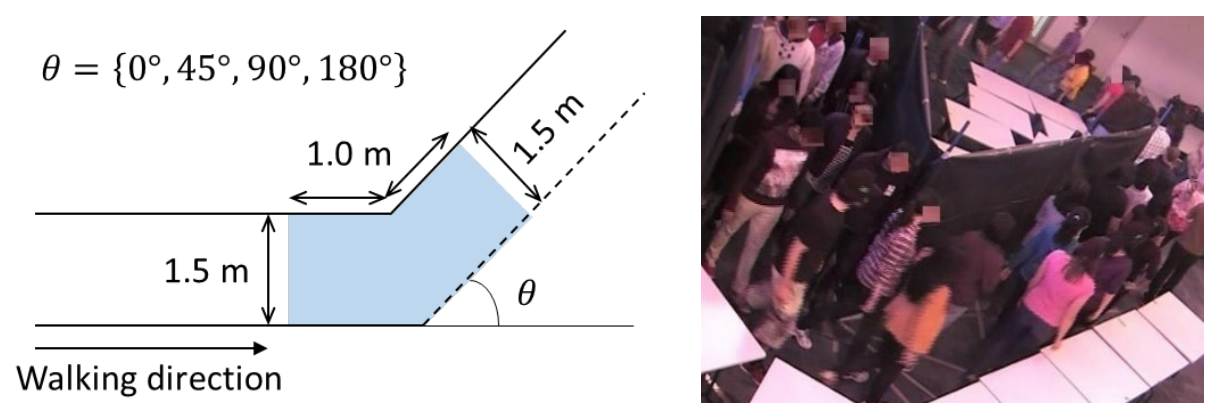

Fig. 1 Experiment setup (left) and one snapshot (right).

\section{Measurement Method}

This study adopted Voronoi diagram method to calculate pedestrian traffic variables since it was reported to be able to reduce the density scatter and at the same time improve the resolution of data with higher sampling rate [3]. The basic idea of this method is that at each frame or during a short enough time period, a Voronoi cell area, $A_{i}$, can be obtained for each person $i$. Then, the density and velocity for a measurement area can be defined as:

$$
\begin{gathered}
\rho_{x y}=1 / A_{i} \text { and } v_{x y}=\left\|\vec{v}_{i}(t)\right\| \quad \text { if }(x, y) \in A_{i} \\
\langle\rho\rangle_{v}=\frac{\iint \rho_{x y} d x d y}{|A|},\langle v\rangle_{v}=\frac{\iint v_{x y} d x d y}{|A|}
\end{gathered}
$$

Where $\rho_{x y}$ and $v_{x y}$ are the density and speed within voronoi cell $A_{i}$ respectively. $|A|$ is the measurement area and in this study it was specified as $1 \mathrm{~m}$ range from the inner turning point at both sides shown as blue shadowed region in Fig. $1 . \vec{v}_{i}(t)$ is the instantaneous speed of person $i$ and it is calculated using the displacement during adjacent time instants from trajectory data. Finally, the flow is obtained by multiplying the Voronoi density $\langle\rho\rangle_{v}$ and speed $\langle v\rangle_{v}$.

\section{Flow Characteristics}

By applying the Voronoi diagram method, the density-flow relations for different angles and desired speeds were derived as shown in Fig. 2. Firstly, it can be seen that only the free flow regime of fundamental diagram was observed and therefore, the capacity of the bend cannot be accurately estimated. However, still it can be observed that the maximum flow for running scenarios $(\approx 2.5 \mathrm{ped} / \mathrm{m} / \mathrm{s})$ was significantly higher than that for walking scenarios $(\approx 1.5 \mathrm{ped} / \mathrm{m} / \mathrm{s})$. This indicates that slow speed running could probably increase the efficiency of bends by around $60 \%$ compared to normal speed walking. Secondly, the regression lines without intercept were drawn to compare the impact of turning angle on the moving speed. Since the slope of the regression line can be approximately considered as the average moving speed of pedestrians, the change rate of slope therefore represents the influence of angle. It can be calculated that from $0^{\circ}$ to $90^{\circ}$, the slope dropped about $20 \%$ for each 45 degrees under running scenarios while only $11 \%$ for walking scenarios. Also from $0^{\circ}$ to $180^{\circ}$, the slope decreased $50 \%$ for running but $35 \%$ for walking. These differences imply that turning angle has a stronger negative impact on the average moving speed of the crowd under running or evacuation conditions.

Further, we used the criterion of Fruin [4] to divide the congestion levels and separately compared average speed and standard deviation under each level of service as presented in Table 1. It can be observed that the speed under three LOSs didn't show a consistent and significant transition which may suggest that compared to the turning angle, congestion level had a minor impact on the moving speed through bends. On the other hand, for $90^{\circ}$ and $180^{\circ}$ bends, the standard deviations continuously decreased with the deterioration of level of services. This indicates that at right-angle and U-turn corridors, high congestion level would homogenize the moving speed of the crowd by restricting individual's freedom of choosing his or her desired speed. 

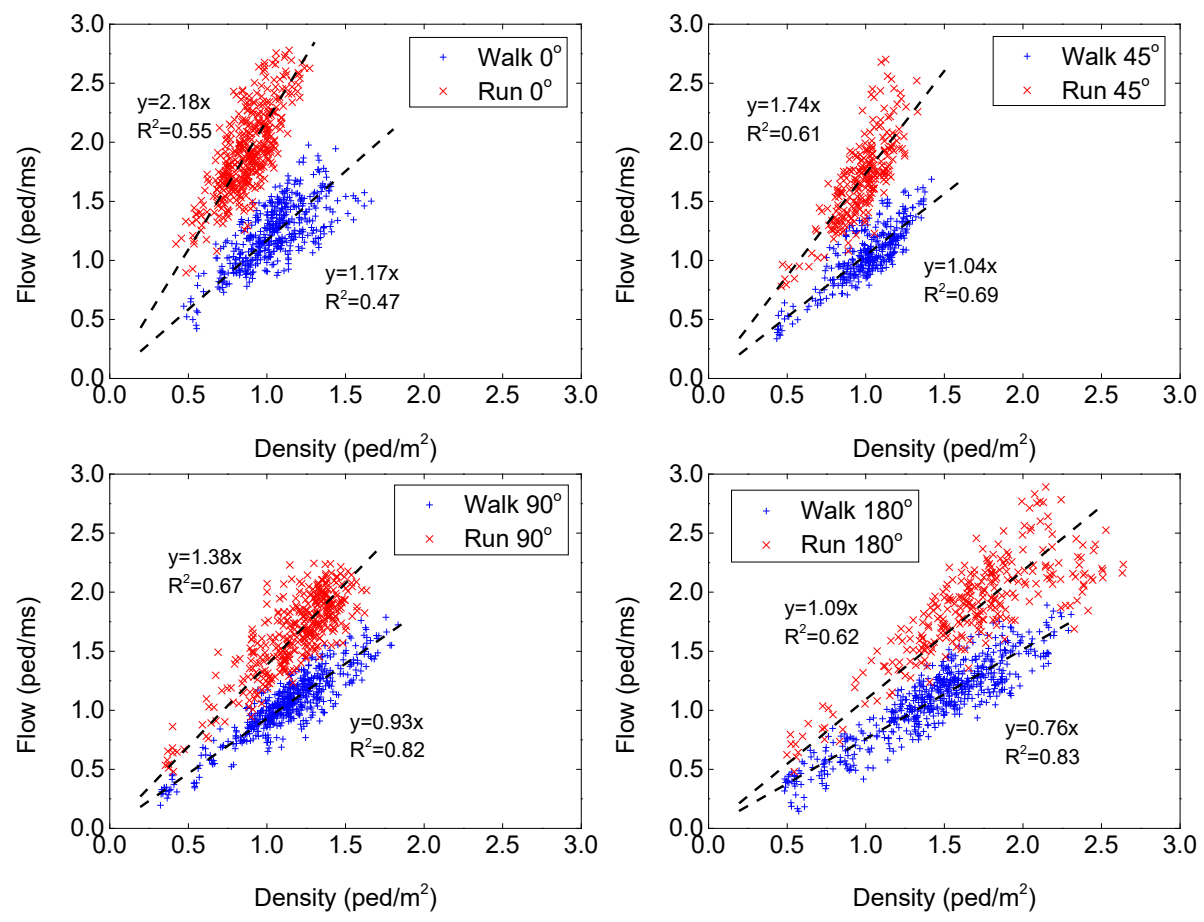

Fig. 2 Comparison of flow-density relations at bends.

Table 1: Average moving speed $(\mathrm{m} / \mathrm{s})$ and its standard deviation $(\mathrm{STDEV}, \mathrm{m} / \mathrm{s})$ under different levels of service.

\begin{tabular}{ccccccccc}
\hline Scenario & Walk $0^{\circ}$ & Walk $45^{\circ}$ & Walk $90^{\circ}$ & Walk $180^{\circ}$ & Run $0^{\circ}$ & Run $45^{\circ}$ & Run $90^{\circ}$ & Run $180^{\circ}$ \\
\hline LOS C & 1.24 & 0.98 & 0.90 & 0.71 & 2.35 & 1.72 & 1.57 & 1.25 \\
(STDEV) & $( \pm 0.22)$ & $( \pm 0.13)$ & $( \pm 0.15)$ & $( \pm 0.20)$ & $( \pm 0.30)$ & $( \pm 0.19)$ & $( \pm 0.27)$ & $( \pm 0.19)$ \\
LOS D & 1.22 & 1.06 & 0.94 & 0.73 & 2.19 & 1.73 & 1.47 & 1.20 \\
$($ STDEV) & $( \pm 0.17)$ & $( \pm 0.15)$ & $( \pm 0.10)$ & $( \pm 0.14)$ & $( \pm 0.29)$ & $( \pm 0.22)$ & $( \pm 0.22)$ & $( \pm 0.17)$ \\
LOS E & 1.15 & 1.04 & 0.93 & 0.76 & 2.15 & 1.76 & 1.37 & 1.12 \\
$($ STDEV) & $( \pm 0.18)$ & $( \pm 0.12)$ & $( \pm 0.09)$ & $( \pm 0.09)$ & $( \pm 0.15)$ & $( \pm 0.23)$ & $( \pm 0.15)$ & $( \pm 0.14)$ \\
\hline
\end{tabular}

\section{Conclusions}

This study quantitatively investigated the flow characteristics of the crowds moving through bends with different turning angles at two desired speed levels. We found that larger turning angle significantly reduced the moving speed under running conditions compared to walking and the maximum flow when pedestrians were walking was around $60 \%$ of that when they were running. On the other hand, compared to turning angle, congestion level at bends was observed to have a minor impact on the moving speed although it cut down the variations of speed by restricting the movement freedom of individuals.

\section{References}

[1] C. Dias, M. Sarvi, N. Shiwakoti, O. Ejtemai and M. Burd, "Examining the Impact of Different Turning Angles on the Collective Egress of Crowds", Journal of Transportation Safety \& Security, vol. 6, no. 2, pp. 167-181, 2014.

[2] C. Dias, M. Sarvi, O. Ejtemai and M. Burd, "Elevated desired speed and change in desired direction: effects on collective pedestrian flow characteristics", Transp Res Rec, vol. 2490, pp. 65-75, 2015.

[3] B. Steffen and A. Seyfried, "Methods for measuring pedestrian density, flow, speed and direction with minimal scatter", Physica A, vol. 389, no. 9, pp. 1902-1910, 2010.

[4] J. Fruin, Pedestrian planning and design. New York: Metropolitan Association of Urban Designers and Environmental Planners, 1971. 